\title{
Misura in tempo reale degli odori negli impianti di depurazione delle acque reflue
}

\author{
Gerardina Erra ${ }^{1}$ \\ ${ }^{1}$ Tecnologie per l'ambiente
}

\begin{abstract}
Le emissioni odorigene rappresentano uno degli aspetti negativi derivanti dall'esercizio delle attività di gestione degli impianti di trattamento ambientali. La possibilità di regolamentare le emissioni odorigene è un problema arduo, legato alla difficoltà di oggettivare la molestia olfattiva, influenzata dalla natura delle sostanze trattate, dalla loro variabilità nel tempo, dalle condizioni meteoclimatiche. Attualmente i metodi di misura e di monitoraggio degli odori sono: sensoriali, analitici e senso-strumentali. Particolare attenzione è rivolta alla misura strumentale con l'applicazione di strumenti multisensoriali, gli IOMS (Instrumental Odour Monitoring System). Gli IOMS rappresentano difatti gli strumenti con il più alto potenziale di sviluppo futuro nelle applicazioni del monitoraggio degli odori in continuo. L'attività di ricerca svolta, presso il laboratorio di ingegneria sanitaria ambientale Seed dell'Università di Salerno, si inserisce nell'ambito delle tematiche della caratterizzazione ed il controllo delle emissioni odorigene con sistemi integrati di tipo strumentale, IOMS. Gli studi si sono focalizzati, in particolare, sulla investigazione degli aspetti di gestione sul lungo termine degli IOMS.
\end{abstract}

\section{Le emissioni odorigene}

Le emissioni odorigene sono generate dall'odore che si manifesta in aria ambiente per la diffusione delle molecole odorigene nella stessa, ad oggi, rappresentano uno degli aspetti più importanti di 
impatto ambientale delle attività antropiche. Esse sono legate alle attività industriali che trattano materiali biodegradabili con trattamenti non efficienti o gestiti in maniera non adeguata in determinate condizioni di temperatura, pressione e condizioni anaerobiche che ne modificano la natura della sostanza rendendola scadente ${ }^{12}$.

Le emissioni odorigene modificano e/o stravolgono del tutto l'equilibrio, le abitudini e i comportamenti di una persona in maniera negativa attraverso sintomi come l'insorgere di mal di testa, disturbo del sonno, perdita dell'appetito, ecc. ${ }^{1}$. Tale aspetto genera una serie di impatti negativi per il territorio stesso con il deprezzamento dei suoli, la svalutazione del bene e la diminuzione della presenza di attività commerciali e turistiche in zona e nei centri limitrofi alla fonte dell'emissione ${ }^{3}$. L'opinione pubblica associa, sempre di più, agli impianti di depurazione, di compostaggio e di discariche controllate emissioni sgradevoli o maleodoranti, per tali motivo vi è un rifiuto alla loro realizzazione prescindendo dal reale impatto. Tale comportamento viene identificato come sindrome NIMBY (Not In My Back Yard, "non nel mio cortile") ${ }^{4}$. Atteggiamento che considera necessario un impianto di depurazioni delle acque reflue (Fig. 1), di un termovalorizzatore ma di non volerlo per gli effetti negativi nel proprio territorio locale. Le proteste e le manifestazioni da parte dei cittadini contro le emissioni odorigene e la realizzazione di strutture necessarie sono aumentate del 22\% nel periodo compreso tra il 2017 ed il 2019. Tra le emissioni odorigene maggiormente percepite vi sono quelle correlate ai letami, solventi, uova marce, bruciato, ammoniaca, immondizia 56 .

\section{Sistemi Strumentali di Monitoraggio degli Odori IOMS}

Il Sistema IOMS, Instrumental Odour Monitoring System, è un dispositivo per il monitoraggio in continuo delle emissioni odorigene e la conseguente valutazione dell'impatto olfattivo. Il sistema è dotato di una cella di misura in cui sono alloggiati sensori diversi, i più utilizzati sono i cosid- 
detti MOS (metal oxide sensor), ma trovano impiego anche sensori elettrochimici (EC), specifici per singoli inquinanti come ammoniaca e acido solfidrico, e a fotoionizzazione (PID), selettivi per composti insaturi (idrocarburi aromatici, olefine, composti carbonilici) ${ }^{7}$. 1 sistema IOMS prevedono una fase iniziale, "l'addestramento", ai fini della realizzazione dei modelli di classificazione e quantificazione degli odori da investigare ${ }^{89}$. A tale scopo si prelevano campioni di odore dalle sorgenti da investigare, sottoponendoli alla rilevazione dello IOMS e ad analisi olfattometriche per la determinazione della concentrazione di odore in accordo alla UNI EN 13725:2004 10116 . Una volta acquisiti tutti i campioni si passa alla analisi statistica multivariata dei dati rilevati, per costruire i modelli di classificazione e quantificazione. L'IOMS, installato presso un ricettore sensibile, registra in continuo la presenza di odori, ne riconosce la provenienza e ne fornisce l'intensità in unità olfattometriche. In questo modo, dal rapporto tra il numero di eventi odorigeni e il tempo totale, è possibile ricavare la frequenza degli episodi di odore, che è in relazione con la valutazione quantitativa della molestia olfattiva e quindi con la normale tollerabilità degli esposti ${ }^{12}$. La matrice di sensori simula il funzionamento dei recettori del naso umano mentre il sistema di elaborazione dei dati quello del cervello. L'IOMS è in grado di caratterizzare e memorizzare l'impronta olfattiva di una miscela odorosa senza entrare nel dettaglio della sua composizione chimica ${ }^{1314}$.

Pertanto, se addestrato per un adeguato periodo di tempo, esso può riconoscere un odore già presente nel suo archivio. Il sistema può essere utilizzato per applicazioni rapide di controllo qualità nell' ambito dell'industria chimica, alimentare, e della cosmesi. In campo ambientale trova applicazione in quattro categorie principali ${ }^{15}$ :

1. monitoraggio della qualità dell'aria;

2. monitoraggio della quaità dell'acqua;

3. controllo dei processi e verifica dell'efficienza del sistema di controllo degli odori per la valutazione dell'impatto odorigeno.

Il vantaggio offerto da questa apparecchiatura è l'oggettività del dato che non richiede la presenza 
umana. Il risultato finale è una valutazione complessiva della miscela di odori, non limitata al singolo composto ${ }^{6}$. L'elaborazione dei dati avviene grazie ad algoritmi che permettono di scegliere le informazioni provenienti dai diversi sensori, di assimilare le differenti risposte e, quindi, di istruire il naso elettronico. Questa fase di apprendimento genera un archivio che risulta essere di fondamentale importanza per poter confrontare le miscele gassose oggetto di studio ${ }^{1615}$.

L'applicazione dei sistemi IOMS ai fini del monitoraggio e della qualità dell'aria ha acquistato un importante rilevanza economica e scientifica, arrivando ad avere anche valore legale 17. L'introduzione in commercio di sistemi elettronici elimina gli svantaggi legati alla soggettività del giudizio, cioè la variabilità individuale, e l'adattamento, cioè la diminuzione della sensibilità durante esposizioni prolungate a un odore ${ }^{1819}$. Da una ricerca effettuata si stima che il mercato dei sistemi IOMS registrerà un aumento del 10,37\% nel periodo di previsione 2020-2025. Il mercato dei sistemi di monitoraggio è suddiviso in due categorie, per settore dell'utente finale (alimenti e bevande, chimica e petrolchimica, ambientale, medica e clinica) e geografia. Il Nord America detiene la quata di mercato più significativa, seguito dal mercato Europeo in più rapida crescita ed infine quello Asiatico ${ }^{6}$.

Sono presenti in commercio una vasta gamma di sistemi di monitoraggio, basati su diversi tipi di sensori, elaborazione dei dati e sistemi di riconoscimento dei modelli e/o principi di funzionamento, in modo tale che vengano stabilite le procedure esatte per la verifica dell'idoneità e dell' utilizzo dello strumento ${ }^{14} 6$.

\section{Conclusioni}

Le emissioni odorigene associate alle fasi di esercizio degli impianti di trattamento di ingegneria sanitaria ambientale costituiscono uno degli aspetti negativi più avvertiti dalla popolazione circostante. Sebbene non siano stati dimostrati conseguenze dirette sulla salute dell'uomo rappresentano la prima fonte di lamentele e di potenziali cause, diventando argomento di contesa sia per gli impianti già esistenti, sia per l'insediamento di nuovi impianti. Risulta dunque fondamentale 
caratterizzare ed identificare strumenti e tecnologie di controllo. I sistemi strumentali IOMS, in tal senso, rappresentano un valido strumento con il più alto potenziale sviluppo futuro. L'influenza di problematiche quali il drift ed il disallineamento delle risposte dei segnali fa si che l'attivita di ricerca è in corso. L'introduzione di elementi di correzioni sono stati analizzati ed introdotti al sistema, al fine di migliorare l'affidabilità della misura, la stabilità del segnale e garantire la sua ripetibilità nel tempo.

\section{References}

1.Zarra, T., Naddeo, V., Belgiorno, V., Reiser, M. \& Kranert, M. Instrumental characterization of odour: a combination of olfactory and analytical methods. Water Science and Technology 59, 1603-1609 (2009).

2.Zarra, T., Naddeo, V., Belgiorno, V., Reiser, M. \& Kranert, M. Odour monitoring of small wastewater treatment plant located in sensitive environment. Water Science and Technology 58, 89-94 (2008).

3.Gostelow, P., Parsons, S. A. \& Stuetz, R. M. Odour measurements for sewage treatment works. Water Research 35, 579-597 (2001).

4.Zarra, T., Naddeo, V. \& Belgiorno, V. Odour measurement in wastewater treatment plant using both European and Japanese standardized methods: correlation and comparison study. Issue $4 \mathbf{1 8}$, 728-733 (2016).

5.T.Zarra et al.. Environmental odour monitoring by electronic nose. Global NEST Journal Vol 20, No X, pp XX-XX, (2018).

6.Zarra, T., G.Galang, M., Ballesteros, F., Belgiorno, V. \& Naddeo, V. Environmental odour man- 
agement by artificial neural network - A review. Environment International 133, 105189 (2019).

7.Giuliani, S., Zarra, T., Naddeo, V. \& Belgiorno, V. A novel tool for odor emission assessment in wastewater treatment plant. Desalination and Water Treatment 55, 712-717 (2014).

8.Naddeo, V. et al.. Odour measurement in wastewater treatment plant by a new prototype of e.nose: Correlation and comparison study with reference to both European and Japanese approaches. CHEMICAL ENGINEERING TRANSACTIONS Vol. 54 p. 85-90,, (2016).

9.Viccione, G., Zarra, T., Giuliani, S., Naddeo, V. \& V.Belgiorno. Performance study of e-nose measurement chamber for environmental odour monitoring. Chemical Engineering Transactions vol. 30, p. 109-114, ISSN: 1974-9791, (2012).

10.T.Zarra, Reiser, M., Naddeo, V., V.Belgiorno \& Kranert, V. A comparative and critical evaluation of different sampling materials in the measurement of odour concentration by dynamic olfactometry. Chemical Engineering Transactions Chemical Engineering Transactions, (2012).

11.Viccione, G., Spiniello, D., Zarra, T. \& Naddeo, V. Fluid dynamic simulation of odour measurement chamber. Chemical Engineering Transactions ISSN: 1974-9791, (2014).

12.Zarra, T. \& Belgiorno, V. Characterization of odours emitted by liquid waste treatment plants (LWTPs). Issue 4 18, 721-727 (2016).

13.Naddeo, V., Zarra, T., G.Oliva, Chiavola, A. \& Vivarelli, A. Environmental odour impact assessment of landfill expansion scenarios: Case study of Borgo Montello (Italy). CHEMICAL ENGINEERING TRANSACTIONS vol. 54, p. 73-78, ISSN: 2283-9216, doi: 10.3303/CET1654013, (2016).

14.Giuliani, S., Zarra, T., Naddeo, V. \& V.Belgiorno. Measurement of odour emission capacity in wastewater treatment plants by multisensor array system. Environmental Engineering and Management Journal vol. 12 (S11), pp. 173-176, ISSN: 1582-9596,, (2013). 
15.Belgiorno, V., V.Naddeo \& Zarra, T. Odour Impact Assessment Handbook. (John Wiley \& Sons Inc., 2012). doi:10.1002/9781118481264.

16.S.Giuliani et al.. An alternative approach of the e-nose training phase in odour impact assessment. Chemical Engineering Transactions vol. 30, p. 139-144, ISSN: 1974-9791, (2012).

17.Villecco, F., Zarra, T. \& Naddeo, V. An Electronic Nose Based On Fuzzy Logic To The Environmental Monitoring Of The Industrial Plants. FLOGEN Vol.4: Meech Intl. Symp. On Mining, ISBN 978-1-987820-11-9, ISSN 2291-1200, (2015).

18.T.Zarra, V.Naddeo \& Belgiorno, V. Characterization of odours emitted by liquid waste treatment plants. Global NEST Journal Vol 18, No 4, pp 721-727, (2016).

19.Naddeo, V. et al.. Odour emissions characterization for impact prediction in anaerobic-aerobic integrated treatment plants of municipal solid waste.. CHEMICAL ENGINEERING TRANSACTIONS vol. 54, p. 91-96, ISSN: 2283-9216, doi: 10.3303/CET1654016, (2016). 
Figure Captions

Figure 1. Fase di sedimentazione secondaria nel trattamento delle acque reflue. 
Figures

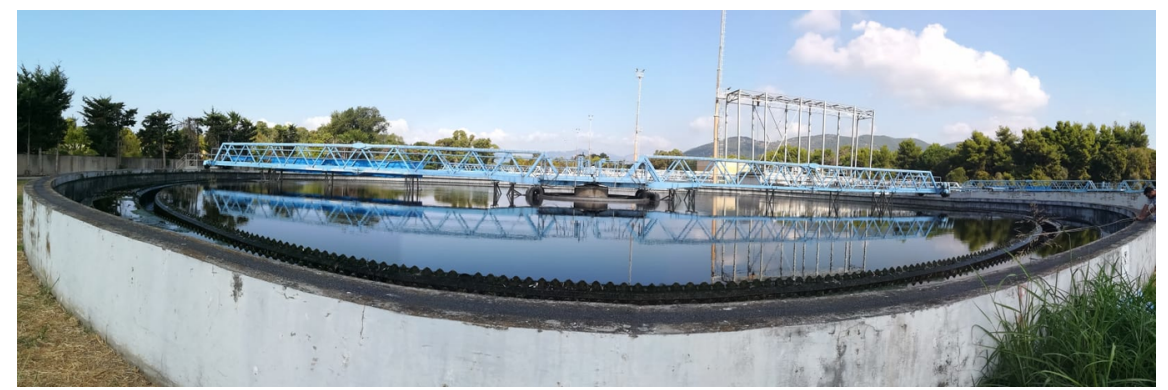

Figure 1: Fase di sedimentazione secondaria nel trattamento delle acque reflue. 